\title{
Correlative Microscopy in Characterization of Polycrystalline YAG Fibers
}

\author{
Kathleen Shugart ${ }^{1,2}$,Hyun Jun Kim ${ }^{1,2}$, Randall Hay ${ }^{1,}$ Benjamin Griffin ${ }^{1,2}$, Ali Kadhim ${ }^{1,2}$ \\ 1. Air Force Research Laboratory, Wright-Patterson AFB, USA \\ 2. UES, Inc. Dayton OH, USA
}

Current research into fibers for laser applications is ongoing due to the ability of fibers to eliminate freespace optics, along with being smaller, lighter and more powerful than slab lasers [1]. Current fiber lasers use silica glass cores, however mechanical failure and thermal lensing are significant problems. Yttrium aluminum garnet $\left(\mathrm{Y}_{3} \mathrm{Al}_{5} \mathrm{O}_{12}, \mathrm{YAG}\right)$ is currently being considered as a host material for highenergy fiber lasers as it has much higher thermal conductivity, a higher laser damage threshold, a lower peak stimulated Brillouin Scattering, and does not photodarken. Researchers have produced both single crystal and polycrystalline YAG fibers, with a variety of cladding materials; the processing steps have evolved to improve fiber quality and minimize optical propagation losses [2]. The fibers used in this work were prepared using commercially available YAG powder (Nanocerox, Ann Arbor, MI), Methocel $^{\mathrm{TM}}$ as a binder (E4M grade, Dow, Midland, MI), Glycerol as a plasticizer (Sigma-Aldrich, St. Louis, MO), and deionized water. The powder was heat treated to remove organic contaminants and then powder ball milled to de-agglomerate. Any ball milling impurities were separated by centrifugation. The mixture was extruded using $20-35 \mathrm{MPa}$ through a $50 \mu \mathrm{m}$ diameter nozzle and dried overnight. These were then burned out at $600^{\circ} \mathrm{C}$ and densified between $1500-1750^{\circ} \mathrm{C}$ [3].

Propagation losses for each fiber were measured by aligning a bare single-mode silica fiber to the input of the fiber under test, and illuminating at a wavelength of $1060 \mathrm{~nm}$ using a fiber-coupled solid-state laser. While illuminated, images of the fibers were collected using a near-infrared-enhanced CCD camera coupled to a 10X microscope objective. Images were collected and stitched together along the length of the fiber. Due to the rough nature of the fiber, light scattered out in all directions as the optical mode travelled down the length of the fiber and was collected on the camera, giving the appearance that the fiber was glowing. Particularly bright hot spots in the camera image indicate high scattering regions in the fiber.

Previous microstructural characterization of these fibers includes cross-sectional scanning electron imaging, transmission electron imaging, and energy dispersive spectroscopy. However, these methods do not allow for a localized inspection of the areas showing greatest scattering. In order to improve understanding of the microstructure of these fibers, and its impact on optical propagation, correlative microscopy offers an exciting opportunity. Laser testing, X-ray computed tomography (CT), and dual beam focused ion beam (DB-FIB) 3D cross sectioning are coupled in this work to determine the location and form of flaws causing excessive scattering.

Laser test results for an as-sintered and an as-sintered then polished fiber are shown in Fig 1. The assintered fiber shows much more scatter along the entire length, and the laser did not propagate as far down the fiber, as it did in the polished sample. This is believed to be due to the increased surface roughness of the as-sintered $(\mathrm{RMS}=0.07 \mu \mathrm{m})$ as compared to the polished $(\mathrm{RMS}=0.03 \mu \mathrm{m})$. Both fibers also show hot spots where increased scattering is occurring. The aim of this work was to determine if a microstructural feature (secondary phase, pore, etc) is responsible for these hot spots. DB-FIB operated as a 3D cross sectioning and imaging tool was identified as the appropriate methodology to answer this 
question. However, image processing of the laser test results provides a resolution of hot spot location to no less than a $300 \mu \mathrm{m}$ region, which is unconducive to locating a specific area to mill, where the largest possible cut is $400 \mu \mathrm{m}$ long.

To correlate between the laser test data and locations in the DB-FIB, X-ray CT was used. Low resolution scans were performed along an $8.3 \mathrm{~mm}$ length of fiber known to contain several flaws according to laser testing. These scans identified two areas of interest in the fiber for high $(\sim 700 \mathrm{~nm})$ resolution scans. The first area was identified as a flaw in the glass cladding applied to the fiber to facilitate waveguiding and optical characterization. The second area, shown in Fig. 2, exhibited a large flaw in the fiber, close to the fiber/cladding interface, along with a series of smaller flaws located near the center of the fiber. The CT scan indicated that the flaws were slightly denser than air but less dense than the fiber. This second region was chosen for further analysis. Due to the high precision on the Versa stage, it was possible to accurately locate the same area in the DB-FIB as measured with CT. It was then feasible to mill an area of the fiber known to contain a flaw. Fig 3 shows several slices performed with the DB-FIB, resulting in an image of the $5 \mu \mathrm{m}$ flaw shown in the CT scan. This flaw was identified as a pore in the fiber (Fig 4.)

Correlative microscopy, combing laser testing, X-ray CT scans and DB-FIB, resulted in a stronger understanding of the microstructure of these fibers. Further work will continue analyzing other areas in fibers, identified first with laser testing, to determine if hot spots are caused by pores only, or a variety of microstructural impurities.

References:

[1] M. Ostermeyer, A. Straesser, Opt. Commun. 274 (2007) p.422-428.

[2] H.J. Kim, et al, J. Euro. Cer. Soc. 35 (2015), p.4251-4258

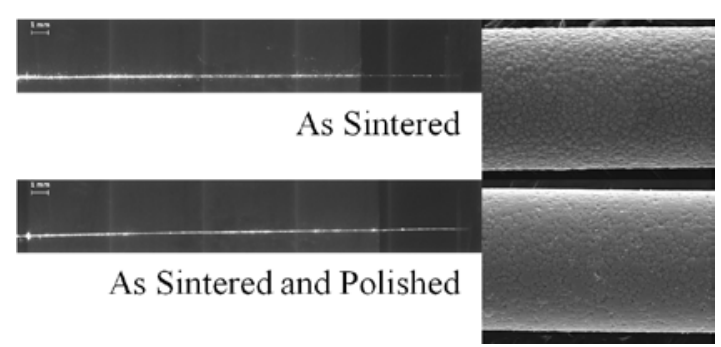

Figure 1. As sintered fiber and laser test results (top); as sintered and then surface polished fiber and laser test results (bottom).

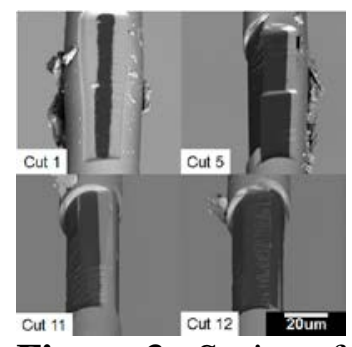

Figure 3. Series of FIB cuts to remove the glass cladding. Flaw is revealed in Cut 12.

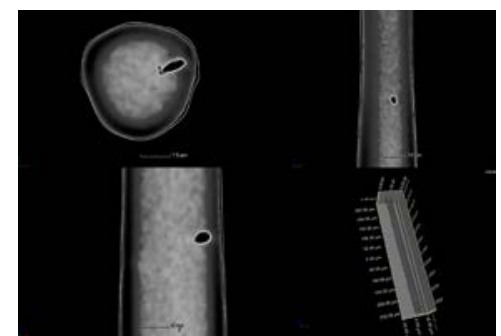

Figure 2. X-ray CT scan of $600 \mu \mathrm{m}$ length of polished fiber. A large $(5 \mu \mathrm{m})$ flaw is seen near the fiber/cladding interface.

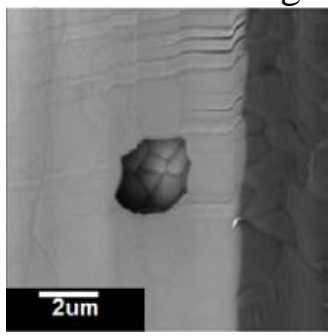

Figure 4. Pore revealed in fiber using DB-FIB, after location was identified using X-ray CT. 